\title{
Downregulation of Scar Fibroblasts by Antineoplastic Drugs: A Potential Treatment for Fibroproliferative Disorders*
}

\author{
M. Georgina Uberti1, Yvonne N. Pierpont1,2\#, Rajat Bhalla',2, Karan Desai1,2, \\ Martin C. Robson'2, Wyatt G. Payne ${ }^{1,2}$ \\ ${ }^{1}$ Institute for Tissue Regeneration, Repair, \& Rehabilitation, Bay Pines V.A. Healthcare System, Bay Pines, USA \\ ${ }^{2}$ Division of Plastic Surgery, University of South Florida, Tampa, USA \\ Email: "yvonne.pierpont@yahoo.com
}

Received 8 May 2016; accepted 10 June 2016; published 13 June 2016

Copyright (C) 2016 by authors and Scientific Research Publishing Inc. This work is licensed under the Creative Commons Attribution International License (CC BY). http://creativecommons.org/licenses/by/4.0/

(c) (i) Open Access

\section{Abstract}

The various fibroproliferative disorders affecting humans have in common excess fibroblast activity and persistent overexpression or dysregulated activity of transforming growth factor beta (TGF- $\beta$ ). Cancer has many similar characteristics. Antineoplastic drugs can downregulate fibroblast activity and cytokine growth factors. This study evaluates the effect of six antineoplastic drugs on keloid and Dupuytren's disease fibroblasts. Keloid, normal scar, Dupuytren's affected palmar fascia, and normal palmar fascia fibroblasts were grown and seeded into Fibroblast Populated Collagen Lattices (FPCLs). The FPCLs were treated with one of six antineoplastic drugs or left untreated as controls. At 7 days, supernatants were extracted from all FPCLs and assayed for expression of Transforming Growth Factor beta (TGF)- $\beta_{1}$ and TGF- $\beta_{2}$. All six antineoplastic drugs significantly inhibited FPCL contraction in both fibroproliferative conditions compared with the untreated controls $(p<0.05)$. Similarly, TGF- $\beta_{1}$ and TGF- $\beta_{2}$ expression was downregulated in the supernatants of all FPCLs by the drug exposure. Cytotoxicity did not occur in these studies and was not the reason for the results. Although antineoplastic drugs can have significant side effects when given systemically, these results may be minimized when given to small areas involved in fibroproliferative scarring or when given topically or intralesionally. These in vitro results suggest that antineoplastic drugs may have a utility for treating various fibroproliferative disorders and warrant further investigation.

\footnotetext{
"Presented in part at: The American Society of Plastic Surgeons Annual Meeting, New Orleans, LA, October 30, 2012; The American Association of Plastic Surgeons 91st Annual Meeting and Symposia, San Francisco, CA, April 15, 2012; The John A. Boswick, MD Burn and Wound Symposium, Maui Hawaii, February 6, 2012.

"Corresponding author. 


\section{Keywords}

\section{Scar Fibroblasts, Antineoplastic Drugs, Fibroproliferative Disorders, Dupuytren's Disease, Keloid}

\section{Introduction}

Various fibroproliferative disorders appear to have similar pathophysiologic features. These disorders such as hypertrophic scar, keloid, rhinophyma, periprosthetic breast capsules, and Dupuytren's contracture have in common active fibroblast activity and overexpression or increased reactivity to the fibrogenic isoforms of Transforming Growth Factor beta (TGF- $\beta$ ) [1]-[8].

Other fibrotic conditions such as lung fibrosis, cirrhosis, glomerulonephritis, and scleroderma are also related to persistent overexpression or dysregulated activation of the cytokine TGF- $\beta$ [1] [2] [9]-[11].

Agents that tend to downregulate fibroblast activity and/or downregulate, abrogate, or neutralize the fibrogenic isoforms of TGF- $\beta$ (TGF- $\beta_{1}$ and TGF- $\beta_{2}$ ) have been suggested to treat some or all of the fibroproliferative disorders discussed. These agents include neutralizing antibodies to TGF- $\beta_{1}$ and TGF- $\beta_{2}$, the non-fibrotic isoform TGF- $\beta_{3}$, interferon gamma, Interferon alpha-2b, mannose-6-phosphate, the decorins, imiquimod, and N-AcetyloL-Cysteine (NAC) [1] [2]. All of these have shown some degree of success in in vitro or animal models.

Tamoxifen, a synthetic nonsteroidal antiestrogen has been shown to be effective in the treatment of abnormal proliferative healing disorders such as retroperitoneal fibrosis and desmoid tumors [12]-[14]. Tamoxifen has also been used to downregulate fibroblast activity and TGF- $\beta_{2}$ expression in Fibroblast-Populated Collagen Lattices (FPCLs) composed of fibroblasts from Dupuytren's disease patients or patients with rhinophyma [15] [16]. Tamoxifen has been demonstrated to have multiple effects including altered ribonucleic acid (RNA) transcription, decreased cellular proliferation, delay or arrest of the cells in the G1 phase of the cell cycle, and interference with several growth factors such as TGF- $\beta$ and insulin-like growth factor [17]-[21]. These attributes and tamoxifen's antiestrogen effects have made it useful as an antineoplastic drug for breast cancer.

If an antineoplastic drug such as tamoxifen is effective against fibroproliferative disorders, it can be postulated that other antineoplastic or antimetabolite drugs may also be effective. In Dvorak's classical article, "Tumors: Wounds that Do Not Heal," he outlined the similarities between tumor stroma generation and wound healing [22]. He discussed six steps in the pathophysiology of cancer formation and wound healing that are identical. Since in excessive healing or proliferative scarring the equilibrium point between collagen deposition and lysis is never reached and wound repair processes seem to continue without an apparent turn-off switch, the fibroproliferative disorders are quite similar to neoplasms. Therefore, the purpose of these studies was to evaluate the downregulation of fibroblast activity and TGF- $\beta_{1}$ and TGF- $\beta_{2}$ expression in FPCLs populated with keloid fibroblasts or fibroblasts harvested from patients with Dupuytren's disease when treated with topical antineoplastic agents. This research seeks to differentiate between potential antineoplastic modalities to decrease fibroblast function while allowing for adequate viability to decrease abnormal scar formation. This will provide a basis of focus for future studies in abnormal scar formation treatment.

\section{Materials and Methods}

The use of all human tissue specimens was performed under the Bay Pines VA Healthcare System Institutional Review Board (IRB) knowledge and approval. Necessary and appropriate consent was obtained from each patient.

Since the fibroblast has been implicated in fibrosing disorders, the Fibroblast-Populated Collagen Lattice (FPCL) has proved useful for understanding these conditions. The methodology for the FPCL has been reported and is divided into four steps: preparation of the fibroblast cultures, preparation of the collagen lattices, assay for gel contraction, and immunoassay of the supernatant for fibrogenic isoforms of TGF- $\beta$ [1] [15] [16].

\subsection{Cell Culture}

Tissue samples were obtained at the time of surgery from six patients who underwent excision of keloids. Control normal appearing scar was obtained from six other patients who underwent elective reoperations for which scar excision was indicated. In addition, palmar fascia samples were obtained at the time of surgery from four patients who had partial fasciectomy for treatment of Dupuytren's Disease. Controls for these samples were ob- 
tained from four other patients who underwent elective hand surgery for non-Dupuytren's Disease problems. The specimens were rinsed in $10 \mathrm{ml}$ of Dulbecco's Modified Eagle's Medium (DMEM) (Invitrogen, Carlsbad, CA, USA), supplemented with 10\% fetal bovine serum heat-inactivated (FBS) (HyClone, Logan, UT, USA), penicillin $(100 \mathrm{U} / \mathrm{ml})$, streptomycin $(100 \mu \mathrm{g} / \mathrm{ml})$ (Sigma-Aldrich, Saint Louis, MO), and amphotericin B (25 $\mu \mathrm{g} / \mathrm{ml}$ ) (Gibco BRL, Grand Island, NY), for 20 minutes at room temperature. Each specimen was then minced into pieces smaller than $5 \mathrm{~mm}$ and plated on the surface of a sterile $100 \mathrm{~mm}$ culture dish in complete media (DMEM containing 10\% FBS). Cell cultures were kept at $37^{\circ} \mathrm{C}$ in $5 \% \mathrm{CO}_{2}$ and $95 \%$ air (Thermo Fisher Scientific Inc., Waltham, MA, USA). The cultured cells were subject to their first passage after they had migrated out of the explants and reached confluence. The explants residues were removed and discarded. The sub-confluent cells were washed using $10 \mathrm{ml}$ of phosphate buffered solution (PBS) (Sigma-Aldrich, Saint Louis, MO), and then detached from the dishes using trypsin-ethylenediaminetetraacetic acid (EDTA) (GIBCO, Grand Island, NY, USA) buffer for 3 minutes at $37^{\circ} \mathrm{C}$, followed by tapping of the dishes and the addition of $5 \mathrm{ml}$ of culture medium to neutralize. The cells were centrifuged at $400 \mathrm{~g}$, and then re-suspended in complete media (DMEM-10\% FBS). The cells were sub-cultured and used for experiments between the third and fifth passage.

During the final passage, the cells were resuspended in complete media to the final cell concentration needed for each experiment. A hemacytometer was used for cell counting, and trypan blue (Sigma-Aldrich, Saint Louis, MO), was used to determine cell viability. The remaining cells were stored at $-70^{\circ} \mathrm{C}$ for future use.

\subsection{Fibroblast-Populated Collagen Lattices (FPCLs)}

The collagen lattices were prepared from type I rat tail collagen as recommended by the manufacturer (Upstate Biotechnology, Lake Placid, NY). Prior to adding the collagen, the 24-well cell culture plates (Fisher Scientific, Pittsburgh, PA), were pre-treated by coating the wells with $1 \mathrm{ml}$ of $0.1 \%$ Bovine Serum Albumin (BSA) (SigmaAldrich, Saint Louis, MO) diluted in PBS. The wells were incubated 2 hours at room temperature. After the supernatant was aspirated, the wells were washed with PBS in order to discard any remnants of BSA, and were left to dry for 10 minutes. Following this prior step, $350 \mu \mathrm{l}$ of undiluted collagen $(3.85 \mathrm{mg} / \mathrm{ml}$ ) was then added per well, and was evenly spread. The well plates were exposed to ammonia vapor in a closed chamber for $15 \mathrm{mi}-$ nutes to solidify. The collagen lattices were then overlaid with sterile distilled water, and were incubated at $37^{\circ} \mathrm{C}$ for 15 minutes. This step was repeated four times to remove excess of ammonia. Complete media (DMEM-10\% FBS) was added to replace the final aspirate. To complete polymerization, collagen gel lattices were incubated for $24 \mathrm{~h}$ at $37^{\circ} \mathrm{C}$ in $5 \% \mathrm{CO}_{2}$ and $95 \%$ air.

Fibroblast-Populated Collagen Lattices (FPCLs) were prepared by seeding the collagen lattices with a suspension of keloid fibroblasts, normal scar fibroblasts, Dupuytren's affected palmar fascia fibroblasts, or normal palmar fascia fibroblasts in complete media to achieve a final cell density of $5.5 \times 10^{5}$ cells $/ \mathrm{ml}$ per collagen lattice. FPCLs were performed in duplicates for every condition, and were maintained during $24 \mathrm{~h}$ at $37^{\circ} \mathrm{C}$ in $5 \%$ $\mathrm{CO}_{2}$ and $95 \%$ air.

\subsection{Drug Preparation and Dilution}

\subsubsection{Tamoxifen}

A stock solution of tamoxifen (500 $\mathrm{\mu g} / \mathrm{ml}$ ) was prepared in DMEM plus 1\% of Dimethyl Sulfoxide (DMSO) (Fisher Scientific, Pittsburgh, PA) to dissolve it. For the FPCLs tamoxifen was then further diluted in DMEM to a final concentration of $10 \mu \mathrm{g} / \mathrm{ml}$.

\subsubsection{5-Fluorouracil (5-FU)}

A stock solution of 5-FU (50 mg/ml) was prepared in DMEM. For the FPCLs 5-FU was then further diluted in DMEM to a final concentration of $100 \mu \mathrm{g} / \mathrm{ml}$.

\subsubsection{Paclitaxel}

A stock solution of paclitaxel $(5 \mu \mathrm{g} / \mathrm{ml})$ was prepared in sterile water $(100 \mathrm{ml})$ plus DMSO $(500 \mu \mathrm{l})$ to dissolve it. For the FPCLs paclitaxel was then further diluted in DMEM to a final concentration of $200 \mathrm{ng} / \mathrm{ml}$.

\subsubsection{Mitomycin-C}

A stock solution of mitomycin-C (400 $\mu \mathrm{g} / \mathrm{ml})$ was prepared in DMEM. For the FPCLs mitomycin-C was then further diluted in DMEM to a final concentration of $0.001 \mathrm{ng} / \mathrm{ml}$. 


\subsubsection{Methotrexate}

A stock solution of methotrexate $(25 \mathrm{mg} / \mathrm{ml})$ was prepared in DMEM. For the FPCLs methotrexate was then further diluted in DMEM to a final concentration of $100 \mu \mathrm{g} / \mathrm{ml}$.

\subsubsection{Bleomycin}

A stock solution of bleomycin $(1.5 \mathrm{mg} / \mathrm{ml})$ was prepared in DMEM. For the FPCLs bleomycin was then further diluted in DMEM to a final concentration of $1 \mu \mathrm{g} / \mathrm{ml}$.

All stock solutions were aliquoted and stored at $-80^{\circ} \mathrm{C}$. Final drugs concentrations were chosen because it was the maximum drug concentration that preserved high cytoviability $(>80 \%)$ and allowed continued proliferation of fibroblasts. These doses also proved to maintain fibroblast morphology to remain normal in collagen lattices.

\subsection{Drug Exposure of Fibroblast-Populated Collagen Lattices (FPCLs)}

Drugs were diluted in DMEM to their final concentrations. FPCLs containing keloid fibroblasts, normal scar fibroblasts, Dupuytren's affected palmar fascia, or normal palmar fascia were then exposed to each drug, or were left untreated as controls (DMEM). Immediately after drug exposure, the untethered FPCLs were then gently released from the edges of the wells using an 18-gauge needle, to float freely and unimpeded from contracting. One hour later, each condition was scanned, and this was considered day 0. The FPCLs were exposed to the drugs during three consecutive days. On the third day, each FPCL was washed three times with $2 \mathrm{ml}$ of PBS to ensure that the amount of residual drug was minimal. The FPCLs were then re-suspended in $1 \mathrm{ml}$ of complete media (DMEM and $10 \% \mathrm{FBS}$ ), and incubated for four more days post-treatment at $37^{\circ} \mathrm{C}$ in $5 \% \mathrm{CO}_{2}$ and $95 \%$ air. All supernatants were collected on the seventh day and snap frozen using liquid nitrogen.

\subsection{Gel Contraction Assay}

The FPCLs were incubated at $37^{\circ} \mathrm{C}$ in a humidified atmosphere of $5 \%$ carbon dioxide. The wells were digitally scanned (HP Photosmart C4280) every 24 hours for the first 3 days and on the $7^{\text {th }}$ day. The amount of gel contraction was measured using digital planimetry and Sigma Scan software (Jandel Scientific, Corte Madera, CA). Each collagen gel area measurement was converted to reflect the percentage of area remaining over time and subsequently the percentage of gel contraction.

\subsection{Effect of Drugs on the Viability of Cells}

To test whether the drugs 5-FU (100 $\mu \mathrm{g})$, bleomycin $(1 \mu \mathrm{g})$, mytomicin-C (0.001 ng), tamoxifen $(10 \mu \mathrm{g})$, methotrexate $(100 \mu \mathrm{g})$, paclitaxel (200 ng) produced their effect by inducing cell death; cell number and viability measurements were made on the seventh day, on FPCLs that had been continuously exposed to the corresponding drug concentration during three days, or were left untreated with DMEM as controls. Duplicates of FPCLs were washed three times with PBS (2 ml) and then overlaid with a solution of $1 \mathrm{mg} / \mathrm{ml}$ collagenase type I (Sigma-Aldrich, Saint Louis, MO) in PBS (1 ml per well), and incubated with light agitation during 1 hour at $37^{\circ} \mathrm{C}$. The cell suspension was then centrifuged, and the resultant cell pellet was re-suspended in $500 \mu \mathrm{l}$ of DMEM.

The total cell number of viable cells was counted from each cell suspension twice on a hemocytometer, with the use of the vital stain trypan blue. The nonviable cells were stained blue, and these were not included on the final number obtained. Results were expressed as the mean percentage of viable cell number of the duplicate FPCLs. This experiment was performed at least twice.

\subsubsection{Quantification of TGF- $\beta_{2}$}

The supernatant obtained from the culture medium following completion of the FPCL contraction assay, was harvested on the seventh day, snap frozen in liquid nitrogen and stored at $-80^{\circ} \mathrm{C}$. To activate latent $T G F-\beta_{2}$ to the immunoreactive form, the samples were exposed to $1 \mathrm{~N} \mathrm{HCL}(100 \mathrm{ml})$ and incubated at room temperature for ten minutes. Samples were then neutralized using a solution of $1.2 \mathrm{~N} \mathrm{NaOH} / 0.5 \mathrm{M}$ HEPES (25 $\mu \mathrm{l})$, followed by addition of $800 \mu \mathrm{l}$ Calibrator Diluent RD5I. To evaluate the concentration of TGF- $\beta_{2}$, samples were assayed immediately using a Quantikine human TGF- $\beta_{2}$ immunoassay (R\&D Systems, Minneapolis, MN).

Briefly, assay dilutent RD1-17 (100 $\mu \mathrm{l})$ was added to the wells of a 96-well microtiter plate, that was precoated with a mouse monoclonal antibody against $T G F-\beta_{2}$. Recombinant human standards for $T G F-\beta_{2}$ (0 to 200 $\mathrm{pg} / \mathrm{ml}$ ), were prepared according to the manufacturer's instructions. Activated samples, controls, and standards 
were added to the plate (100 $\mu \mathrm{l}$ per well), covered with adhesive strip provided, and incubated for 2 hours at room temperature. Microtiter plates were aspirated and washed three times using wash buffer $(400 \mu \mathrm{l})$, and then incubated with $200 \mu \mathrm{l}$ of $T G F-\beta_{2}$ conjugate, during two hours at room temperature. After aspiration, followed by three more washes, the plates were then incubated with $200 \mu \mathrm{l}$ of substrate solution for 20 minutes at room temperature protected from light. The reaction was ceased by adding with $50 \mu \mathrm{l}$ of stop solution. The absorbance was measured at $450 \mathrm{~nm}$ on a microplate spectrophotometer (BioTek model ELx 808).

Once the standard curve was complete the unknown concentrations were determined. Each unknown concentration was then multiplied by a factor of 7.8 to correct for the dilution factor. The readings for each standards, samples and controls were averaged, and subtracted the average of the zero standard optical density.

\subsubsection{Quantification of TGF- $\beta_{1}$}

To activate latent $T G F-\beta_{1}$ to the immunoreactive form, the samples were exposed to $1 \mathrm{~N}$ HCL (100 ml) and incubated at room temperature for ten minutes. Samples were then neutralized using a solution of $1.2 \mathrm{~N} \mathrm{NaOH/0.5}$ M HEPES $(20 \mu \mathrm{l})$. To evaluate the concentration of TGF- $\beta_{1}$, samples were assayed immediately using a Quantikine human TGF- $\beta_{1}$ immunoassay (R \& D Systems, Minneapolis, MN).

Briefly, assay diluent RD1-21 (50 $\mathrm{\mu l}$ ) was added to the wells of a 96-well microtiter plate, that was pre-coated with a monoclonal antibody against TGF- $\beta_{1}$. Recombinant human standards for TGF- $\beta_{1}(0$ to $200 \mathrm{pg} / \mathrm{ml})$, were prepared according to the manufacturer's instructions. Activated samples, controls, and standards were added to the plate ( $50 \mu \mathrm{l}$ per well), covered with adhesive strip provided, and incubated for 2 hours at room temperature. Microtiter plates were aspirated and washed three times using wash buffer ( $400 \mu \mathrm{l})$, and then incubated with 100 $\mu \mathrm{l}$ of TGF- $\beta_{1}$ conjugate, during two hours at room temperature. After aspiration, followed by three more washes, the plates were then incubated with $100 \mu \mathrm{l}$ of substrate solution for 20 minutes at room temperature protected from light. The reaction was stopped by adding with $100 \mu \mathrm{l}$ of stop solution. The absorbance was measured at $450 \mathrm{~nm}$ on a microplate spectrophotometer (BioTek model ELx 808).

Once the standard curve was complete the unknown concentrations were determined. Each unknown concentration was then multiplied by a factor of 1.4 to correct for the dilution factor. The readings for each of the standards, samples and controls were averaged, and the average of the zero standard optical density was subtracted. As animal serum that was used in the preparation of the cell culture media (10\% FBS), contains endogenous TGF- $\beta_{1}$, controls used (DMEM supplemented with $10 \% \mathrm{FBS}$ ), determined the baseline concentration of TGF- $\beta_{1}$.

\subsection{Data Analysis}

As stated above, gel contraction assay wells were digitally scanned (HP Photosmart C4280) every 24 hours for the first 3 days and on the $7^{\text {th }}$ day. The amount of gelcontraction was measured using digital planimetry and Sigma Scan software (Jandel Scientific, Corte Madera, CA). Each collagen gel area measurement was converted to reflect the percentage of area remaining over time and subsequently the percentage of gel contraction.

Quantitative assays for quantification of $T G F-\beta_{1} / T G F-\beta_{2}$ : standard curve for unknown concentrations was determined. Each unknown concentration was then multiplied by appropriate factor per assay type to correct for the dilution factor. The readings for each standards, samples and controls were averaged, and subtracted the average of the zero standard optical density.

Quantitative data was then expressed between different groups as defined above as the mean \pm standard deviation. Statistical significance was determined by the unpaired Student t-test in conjunction with a one-way analysis of variance (ANOVA) to determine significant differences among groups. Differences were considered to be significant for $p<0.05$.

\section{Results}

\subsection{FPCL Contraction}

Keloid fibroblasts caused a significantly greater contraction of the FPCL at each measuring point compared with normal scar fibroblasts $(p<0.01$ ) (Figure 1(a)). Fibroblasts from Dupuytren's affected palmar fascia caused a significantly greater degree of contraction of the FPCL compared with the normal control palmar fascia beginning on day $3(p<0.05)$ (Figure $1(b))$.

All of the antineoplastic drugs tested significantly inhibited the fibroblast contraction of both keloid fibroblasts and Dupuytren's fascial fibroblasts compared with the untreated controls $(p<0.01)(p<0.05)$ (Figure 2(a) 


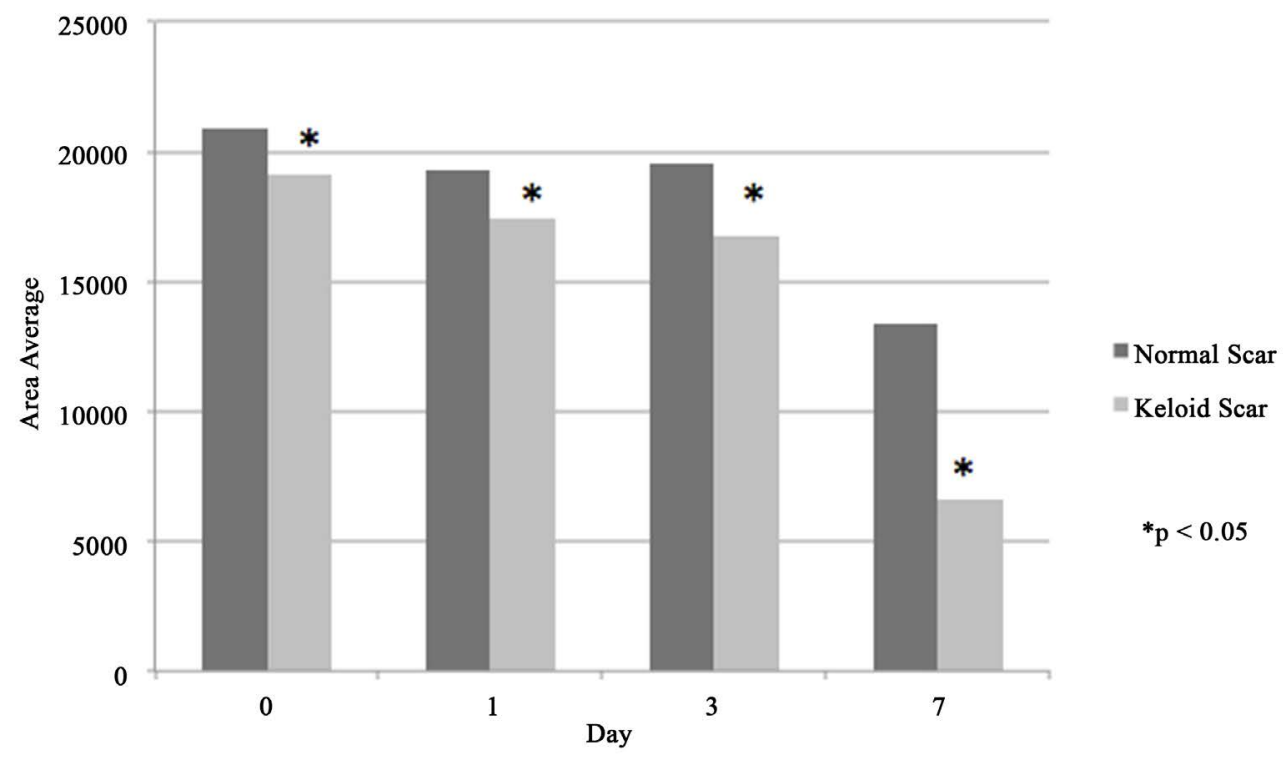

(a)

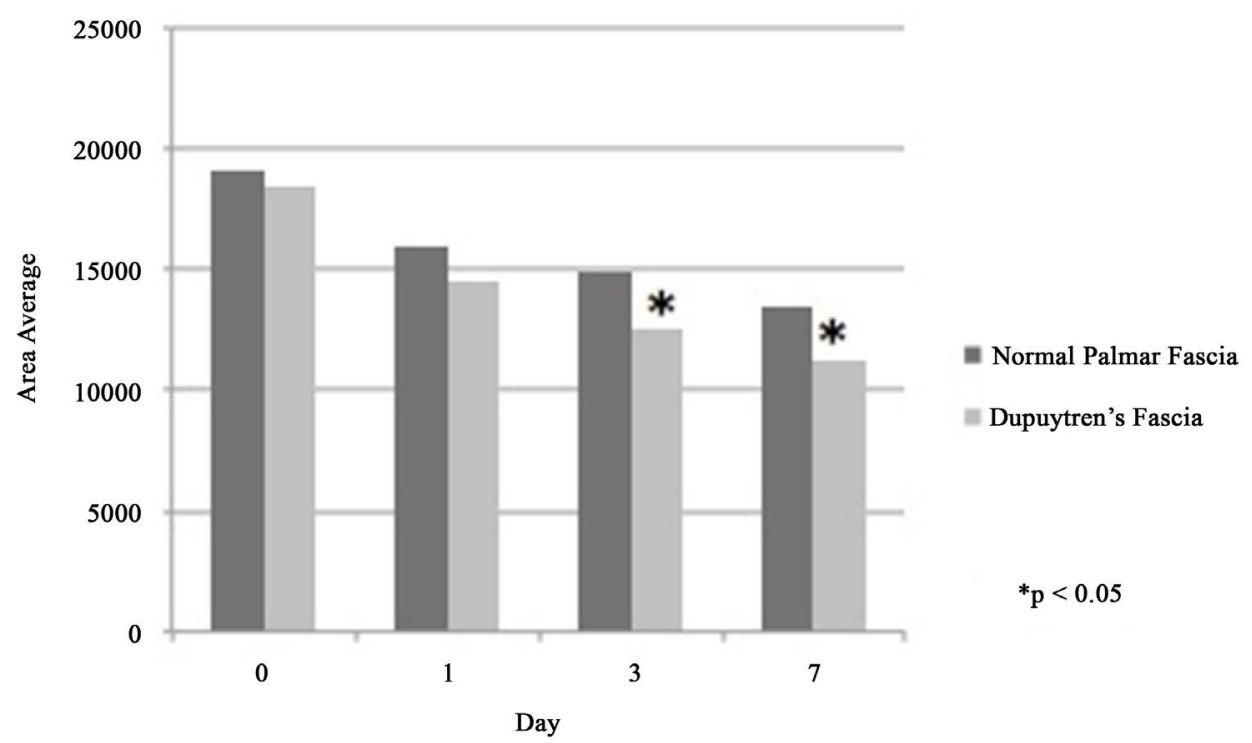

(b)

Figure 1. Fibroblasts from fibroproliferative disorders significantly increased FPCL contraction as seen with (a) keloid fibroblast-seeded lattices $(p<0.01)$ and (b) Dupuytren's affected palmar fascia fibroblast-seeded lattices $(p<0.05)$.

and Figure 2(b)). In the keloid-populated lattices the inhibition was significantly greater when treated with paclitaxel, tamoxifen, and methotrexate compared with the other drug treatments $(p=0.005)$ (Figure 2(a)). There was no statistical differences between paclitaxel, tamoxifen, and methotrexate $(p=0.17)$. Each of the antineoplastic drugs inhibited FPCL contraction in the Dupuytren's affected palmar fascia seeded lattices compared with the untreated controls $(p<0.05)$ (Figure 2(b)). However, there were no statistical significant differences between the various drugs.

The inhibition of FPCL contraction seen with the treatment of antineoplastic drugs in both experiments was not due to fibroblast cytotoxity caused by the drugs. The trypan blue staining showed cell viability both before the lattices were seeded and seven days after treatment with the drugs. Cell number and percent viability were unchanged after the seven days of drug exposure. Fibroblast morphology in the collagen lattices remained normal during the seven days of drug exposure. 


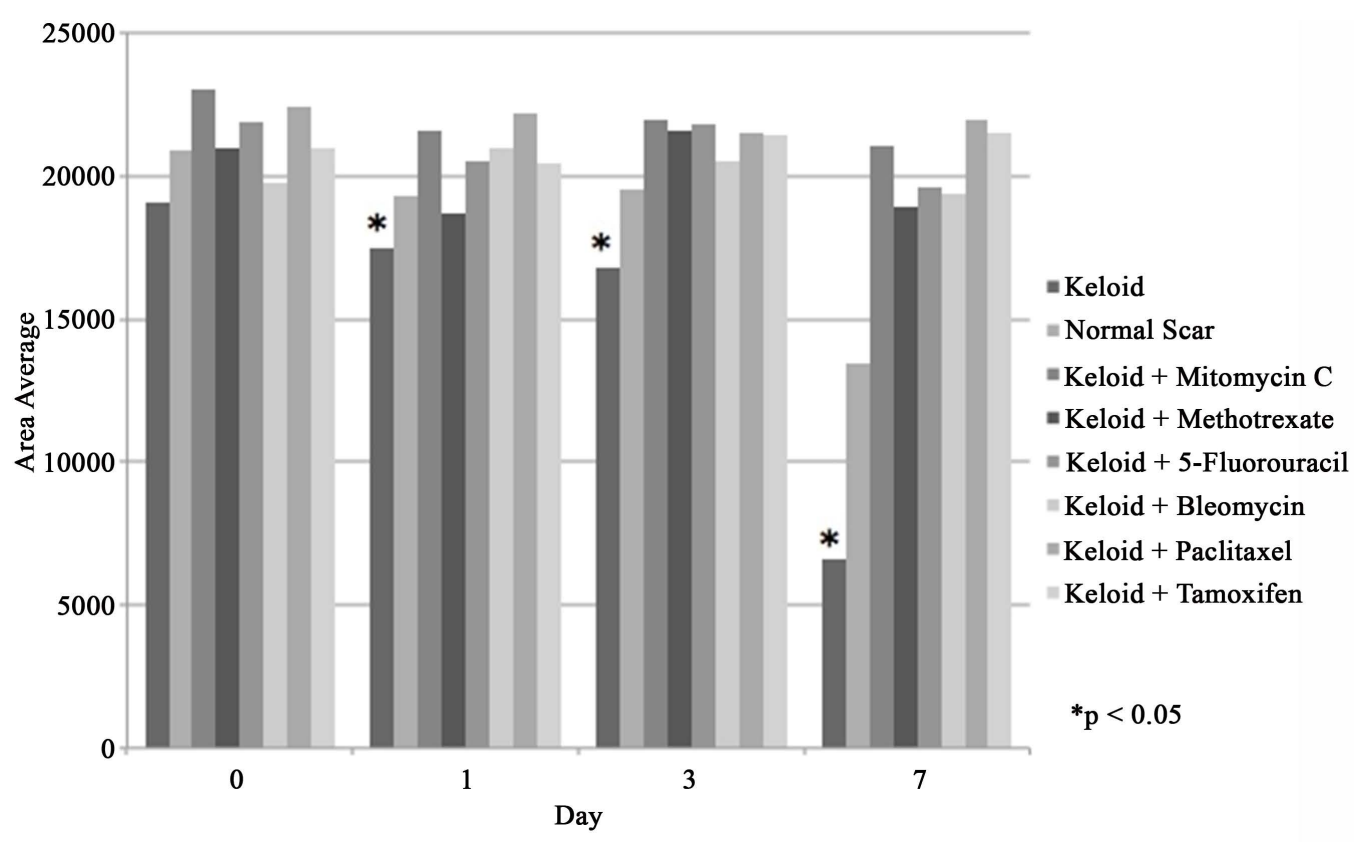

(a)

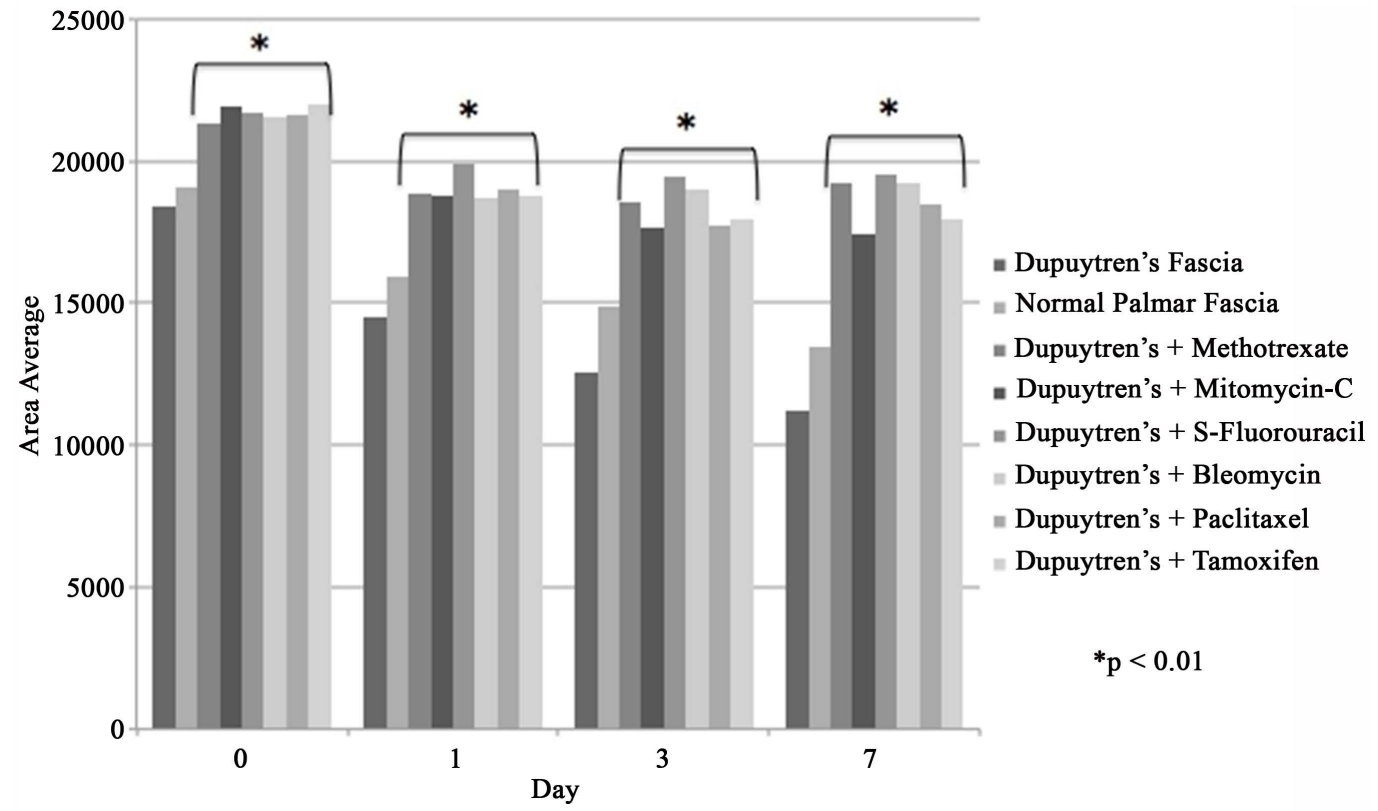

(b)

Figure 2. All six antineoplastic drugs significantly inhibited FPCL contraction as seen with (a) keloid fibroblast-seeded lattices $(p<0.01)$ and (b) Dupuytren's affected palmar fascia fibroblast-seeded lattices $(p<0.05)$. By day 7 paclitaxel, tamoxifen, and methotrexate significantly were more inhibitory than the other three agents in the (a) keloid fibroblast-seeded lattices $(p<0.005$ ), but there were no significant differences among the various drugs in treatment of the (b) Dupuytren's affected palmar fascia fibroblast-seeded lattices.

\subsection{TGF- $\beta_{1}$ and TGF- $\beta_{2}$ Immunoassay}

Expression of TGF- $\beta_{2}$ in the supernatant from untreated FPCLs seeded with keloid fibroblasts was significantly increased compared with those seeded with normal scar control fibroblasts $(p<0.001)$ (Figure 3(a)). Similarly, seeding untreated FPCLs with palmar fascia from Dupuytren disease patients resulted in a supernatant with significantly higher expression of TGF- $\beta_{2}$ than if the untreated FPCLs were seeded with normal control palmar 
Transforming Growth Factor- $\beta_{2}$
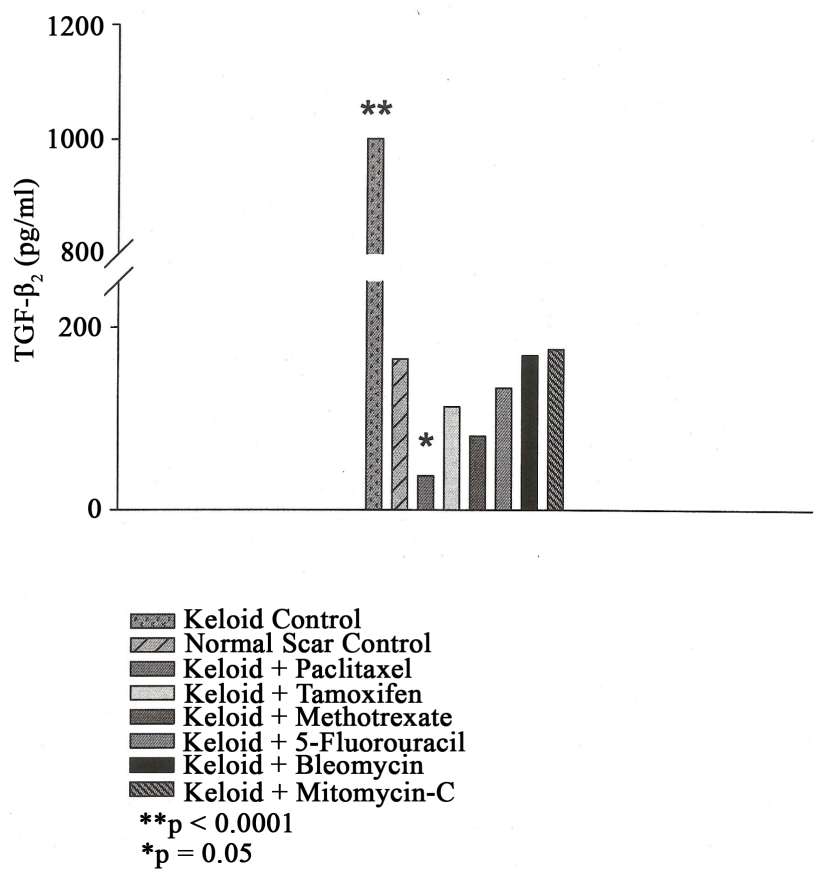

(a)

Transforming Growth Factor- $\beta_{2}$

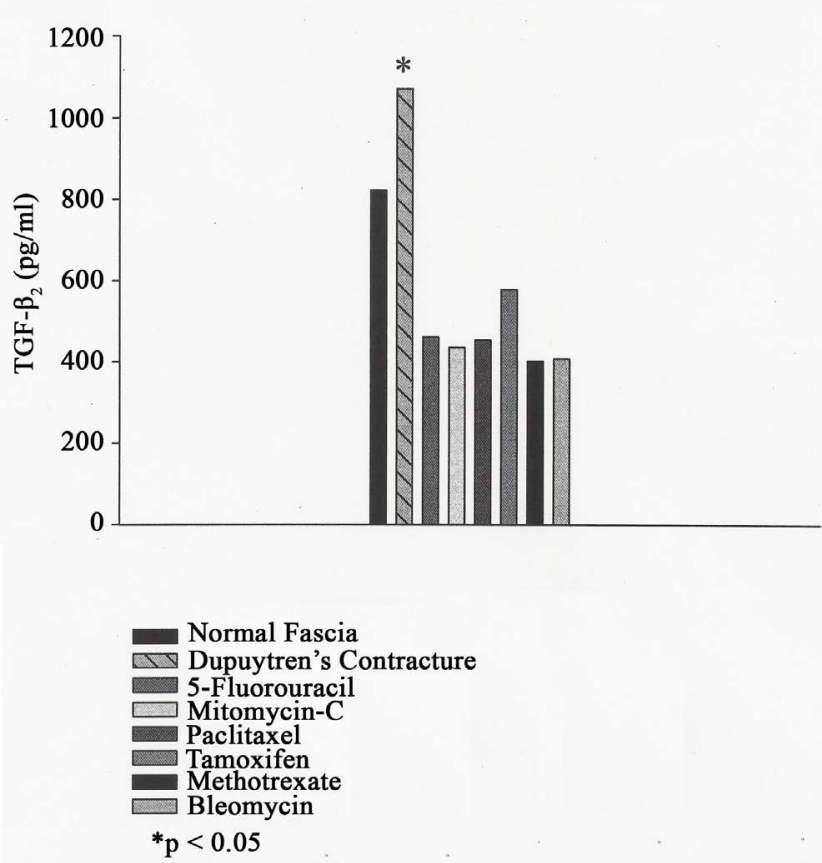

(b)

Figure 3. All six antineoplastic drugs significantly downregulated TGF- $\beta_{2}$ expression in the FPCL supernatant from (a) keloid fibroblast-seeded lattices $(p<0.05)$ and (b) Dupuytren's affected palmar fascia fibroblast-seeded lattices $(p<0.05)$. Downregulation by paclitaxel was significantly greater than the other agents in (a) keloid fibroblast-seeded lattices $(p<0.0001)$. 
fascia fibroblasts $(p<0.005$ ) (Figure 3(b)). Treatment of the FPCLs seeded with keloid fibroblasts with each of the antineoplastic drugs resulted in downregulation of TGF- $\beta_{2}$ expression compared with the untreated keloid FPCL $(p<0.05)$ (Figure 3(a)). Paclitaxel appeared to decrease the TGF- $\beta_{2}$ significantly more than the other drugs $(p<0.001)$. Treatment of FPCLs seeded with Dupuytren's affected palmar fascia fibroblasts by each of the antineoplastic drugs also resulted insignificant downregulation of TGF- $\beta_{2}$ in the supernatants compared with the untreated FPCLs $(p<0.05$ ) (Figure 3(b)). No significant differences between the drugs were observed.

TGF- $\beta_{1}$ expression was not significantly different between supernatants from FPCLs seeded with keloid fibroblasts and fibroblasts from normal scar (Figure 4(a)). However, treatment of the FPCLs with 5 fluorouracil, paclitaxel, tamoxifen, and methotrexate decreased the expression of TGF- $\beta_{1}$ significantly compared with the untreated control FPCLs $(p<0.05)$. Supernatants from FPCLS seeded with either Dupuytren's palmar fascia or control palmar fascia expressed similar levels of TGF- $\beta_{1}$ (Figure 4(b)). Treatment with 5-fluorouracil, mitomycin-c, or methotrexate significantly decreased the TGF- $\beta_{1}$ expression in the supernatants from the Dupuytren's fibroblast-seeded FPCLs compared with the untreated controls $(p<0.05)$ (Figure 4(b)).

\section{Discussion}

Downregulating fibroblast activity and/or downregulating, abrogating, or neutralizing the fibrogenic isoforms of TGF- $\beta$ (TGF- $\beta_{1}$ and TGF- $\beta_{2}$ ) have been suggested to treat some or all of the fibroproliferative disorders discussed. To evaluate the effects of various agents that might accomplish those goals, we have chosen the fibroblast-populated collagen lattice. The FPCL has proven to be an effective in vitro means to evaluate fibroblast activity [1] [23]-[26]. Combining the FPCL contraction with measurements of TGF- $\beta_{2}$ expression in the FPCL supernatant has provided valuable information regarding potential treatment of fibroproliferative disorders [27].

Since Dvorak has discussed the similarities between wound healing and cancer and as the cancer drug tamoxifen has demonstrated the ability to decrease fibrosis and downregulate TGF- $\beta_{2}$ expression, it seems reasonable that other antineoplastic drugs may be effective for treatment of the range of fibroproliferative disorders. A number of in vitro and in vivo studies have demonstrated that certain antiproliferative/antineoplastic agents to decrease abnormal scarring, fibroblast proliferation and contraction, and collagen deposition [28]-[46]. Due to different methodologies, the results from these studies are not directly comparable. Also, none of the reports compared multiple antineoplastic drugs to determine their effects on the rate and extent of fibroblast contraction and their effects on TGF- $\beta$ expression.

Despite the effect of antineoplastic agents on arresting fibroblasts, evidence shows that these cells remain capable of migration and continue to produce regulatory proteins inclusive of growth factors and extracellular matrix (ECM) molecules [40] [43]. In the experiments reported here, the stock solutions were specifically prepared to maintain cytoviability which was proven after 7 days of exposure to the various drugs. In addition to viability, the doses allowed the fibroblast morphology to remain normal in the collagen lattices. This eliminated the possibility that the inhibition to FPCL contraction was due to necrosis of the fibroblasts.

In this study we demonstrated that fibroblasts from keloid scars or from Dupuytren's affected palmar fascia were more active at contracting collagen lattices than their respective control fibroblasts. This observation is the same as has been reported in the literature [1] [15] [47]. After exposure to the stock solutions of tamoxifen, 5fluorouracil, paclitaxel, mitomycin-C, methotrexate, or bleomycin, contraction of the FPCLs decreased whether seeded with keloid fibroblasts or fibroblasts from Dupuytren-affected palmar fascia. In the keloid fibroblastseeded FPCLs, paclitaxel, tamoxifen, and methotrexate were significantly inhibited better than the others; however in the Dupuytren's fibroblast-seeded FPCLS, there were no significant differences among the treatments.

The results from the immunoassays for TGF- $\beta_{1}$ and TGF- $\beta_{2}$ in the fibroproliferativedisorder fibroblast-seeded FPCLscompared with the control fibroblast-seeded FPCLs were consistent with the literature. TGF- $\beta_{2}$ expression is consistently elevated in the fibroproliferative disorders such as keloids and Dupuytren's Disease [1] [2]. The antineoplastic drugs downregulated the expression of TGF- $\beta_{2}$ in the supernatants. Although the expression of TGF- $\beta 1$ was not significantly increased in the fibroproliferative fibroblast-seeded FPCLs, the antineoplastic drugs still decreased the TGF- $\beta_{1}$ expression in supernatants from the FPCLs. It appears that the results of the decreased FPCL contraction may be related to the downregulation of the fibrogenic isoforms of TGF- $\beta$, especially TGF- $\beta_{2}$. This suggests that the effect of the antineoplatic drugs is similar to other treatments which have been tested for treatment of fibroproliferative disorders such as silicone sheeting, neutralizing antibodies to TGF- $\beta$, the decorins, mannose-6-phosphate, and Interferon alpha-2b [2]. 
Transforming Growth Factor- $\beta_{1}$
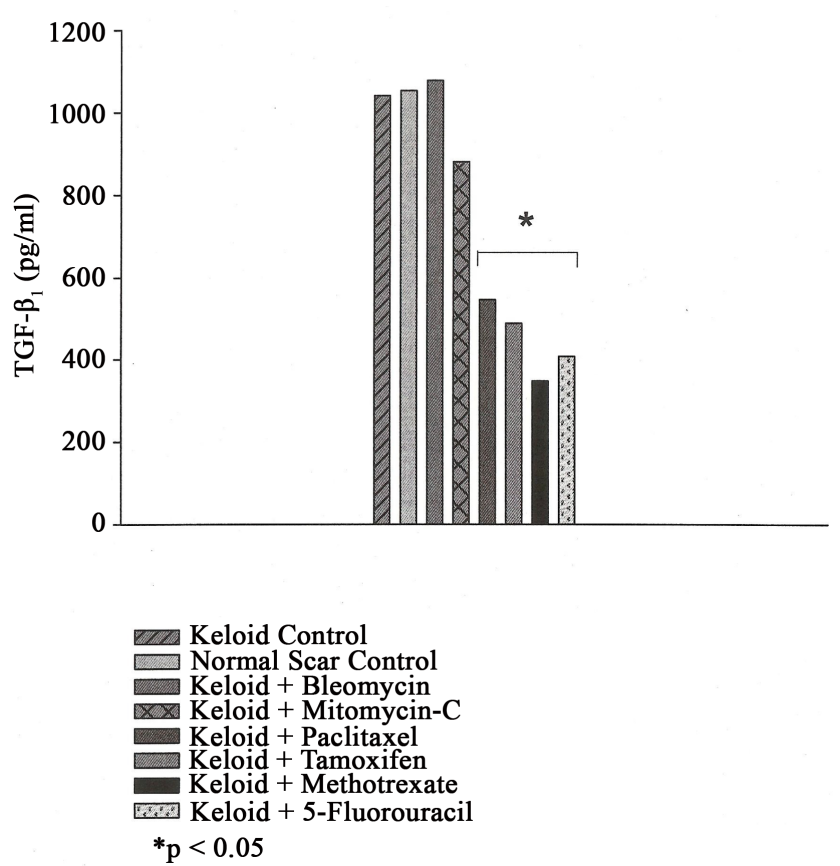

(a)

Transforming Growth Factor- $\beta_{1}$

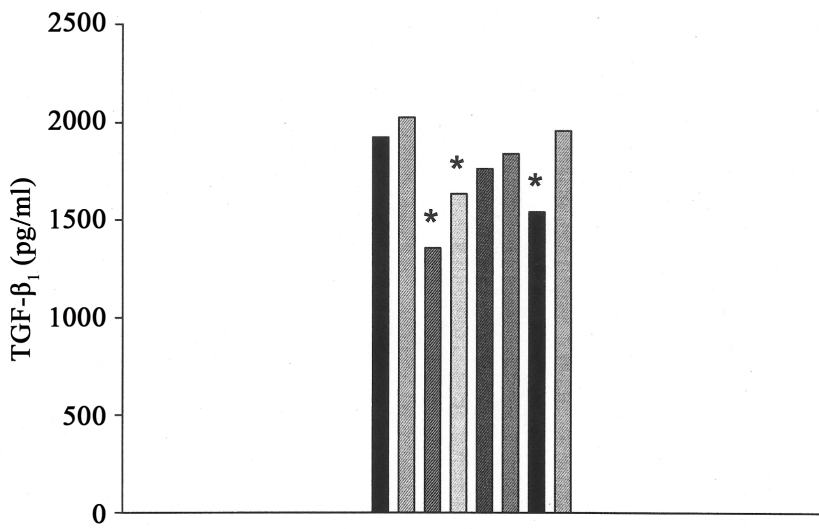

Normal Fasci

Dupuytren's Contracture

-Fluorouracil

Mitomycin-C

Tamoxifen

Bleomycin

${ }^{*} \mathrm{p}<0.05$

(b)

Figure 4. Four of the six antineoplastic agents significantly downregulated TGF- $\beta_{1}$ expression in the FPCL supernatant from (a) keloid fibroblast-seeded lattices $(p<0.05)$; whereas three of the six agents significantly downregulated TGF- $\beta_{1}$ expression in the FPCL supernatant from Dupuytren's affected palmar fascia fibroblast-seeded lattices $(p<0.05)$. 
Proceeding to use antineoplastic drugs for non-malignant conditions requires a great deal of caution. Most of these drugs are only given systemically for treatment of a malignancy. However, for fibroproliferative disorders, possibly they may be applied topically or locally injected into the area of fibrosis. This would keep systemic side effects to a minimum. Despite attempting to minimize doses and side effects, one must be cognizant of the severe side effects of agents used in these experiments. Tamoxifen may be the best-tolerated of the tested drugs. It has been used in topical preparations and in benign fibroproliferative disorders [1]. 5-fluorouracil has been used for intralesional injection, but even with this route of administration has side effects such as pain, skin ulcerations, and hyperpigmentation [48] [49]. Paclitaxel can be associated with bone marrow depression and neurotoxicity but probably not at the doses required for scar conditions. Mitomycin-C also can be associated with bone marrow toxicity and renal failure. One drawback to its use in fibroproliferative disorders is that it causes sloughing of the skin when an intravenous application is extravasated. Methotrexate probably would have to be limited to short-term therapy to avoid alopecia, oral ulcers, and cytopenia [50]. However, the low doses required in the treatment of keloids are considered to be safe. Finally, bleomycin can cause pulmonary, renal, and cutaneous fibrosis at high doses. Doses of bleomycin used for intralesional punctures have shown less than 5\% systemic absorption [32]. Ifthese side effects can be minimized or eliminated, treatment with antineoplastic drugs may be feasible for fibroproliferative disorders that have many of the same characteristics of malignancies.

Limitations of this study include study of only in vitro analysis as well as limited quantity of scar types. Future studies would benefit from incorporating in vivo topical response. Additional in vitro studies of abnormal scar, including hypertrophic scar, rhinophyma, and implant based capsular contraction scar would broaden the scope of fibroblast response in additional abnormal scar formation.

\section{Conclusion}

Fibroprolifereative disorders have many characteristics of malignancies. This study investigated the role of six antineoplastic drugs in downregulating excessive fibroblast activity as measured by FPCL contraction and modulating the expression of the fibrogenic isoforms of TGF- $\beta$. All of the drugs evaluated significantly reduced FPCL contraction and downregulated TGF- $\beta_{1}$ and TGF- $\beta_{2}$ expression in the FPCL supernatants. These findings suggest that further investigation of antineoplastic drugs for the treatment of fibroproliferative disorders may be indicated.

\section{Disclaimer}

This material is the result of work supported with resources and the use of facilities of the Bay Pines VA Healthcare System. Study funded in part by the Plastic Surgery Educational Foundation. The contents of this work do not represent the views of the Department of Veterans Affairs or the United States Government.

\section{References}

[1] Robson, M.C. and Payne, W.G. (2012) Using Laboratory Models to Develop Mechanistic Treatments for Dupuytren's Disease. In: Eaton, C., Seegenschmiedt, M.H., Bayat, A., Gabbiani, G., Werker, P.M. and Wach, W., Eds., Dupuytren's Disease and Related Hyperproliferative Disorders, Springer-Verlag, Berlin, 175-183. http://dx.doi.org/10.1007/978-3-642-22697-7_22

[2] Robson, M.C. (2003) Proliferative Scarring. Surgical Clinics of North America, 83, 557-569. http://dx.doi.org/10.1016/S0039-6109(02)00197-4

[3] Lee, T.Y., Chin, G.S., Kim, W.G.H., Chau, D., Gittes, G.K. and Longaker, M.T. (1999) Expression of Transforming Growth Factor Beta 1, 2, and 3 Proteins in Keloids. Annals of Plastic Surgery, 43, 179-184.

[4] Pu, L.L.Q., Smith, P.D., Payne, W.G., et al. (2000) Overexpression of Transforming Growth Factor Beta-2 and Its Receptor in Rhinophyma: An Alternative Mechanism of Pathobiology. Annals of Plastic Surgery, 45, 515-519. http://dx.doi.org/10.1097/00000637-200045050-00008

[5] Younai, S., Nichter, L.S., Wellisz, T., Reinisch, J., Nimni, M.E. and Tuan, T.L. (1994) Modulation of Collagen Synthesis by Transforming Growth Factor-Beta in Keloid and Hypertrophic SCAR fibroblasts. Annals of Plastic Surgery, 33, 148-151. http://dx.doi.org/10.1097/00000637-199408000-00005

[6] Younai, S., Venter, G., Vu, S., Nichter, L., Nimni, M.E. and Tuan, T.L. (1996) Role of Growth Factors in Scar Contraction: An in Vitro Analysis. Annals of Plastic Surgery, 36, 495-501.

http://dx.doi.org/10.1097/00000637-199605000-00011 
[7] Kuhn, A., Singh, S., Smith, P.D., et al. (2000) Periprosthetic Breast Capsules Contain the Fibrogenic Cytokines TGF$\beta 1$ and TGF- $\beta 2$, Suggesting Possible New Treatment Approaches. Annals of Plastic Surgery, 44, 387-391. http://dx.doi.org/10.1097/00000637-200044040-00006

[8] Kuhn, M.A., Payne, W.G., Kierney, P.C., et al. (2001) Cytokine Manipulation of Explanted Dupuytren’s Affected Human Palmar Fascia. International Journal of Surgical Investigation, 2, 443-456.

[9] Border, W.A. and Rouslahti, E. (1992) Transforming Growth Factor Beta in Disease: The Dark Side of Tissue Repair. Journal of Clinical Investigation, 90, 1-7. http://dx.doi.org/10.1172/JCI115821

[10] Border, W.A. and Noble, N.A. (1994) Transforming Growth Factor Beta in Tissue Fibrosis. New England Journal of Medicine, 331, 1266-1292.

[11] O’Kane, S. and Ferguson, M.W.J. (1997) Transforming Growth Factor Bs and Wound Healing. International Journal of Biochemistry \& Cell Biology, 29, 63-78. http://dx.doi.org/10.1016/S1357-2725(96)00120-3

[12] Owens, L.V., Cance, W.G. and Huth, J.F. (1995) Retroperitoneal Fibrosis Treated with Tamoxifen. Annals of Surgery, 61, 842-844.

[13] Clark, C.P., Vanderpool, D. and Preskill, J.T. (1991) The Response of Retroperitoneal Fibrosis to Tamoxifen. Surgery, 109, 502-506.

[14] Timmons, M.J. (1994) Fibromatosis, Desmoids, Fibroblasts, and Tamoxifen. British Journal of Plastic Surgery, 47, 378-380. http://dx.doi.org/10.1016/0007-1226(94)90100-7

[15] Kuhn, M.A., Wang, X., Payne, W.G., Ko, F. and Robson, M.C. (2002) Tamoxifen Decreases Fibroblast Function and Downregulates TGF Beta-2 in Dupuytren’s Affected Palmar Fascia. Journal of Surgical Research, 103, 146-152. http://dx.doi.org/10.1006/jsre.2001.6350

[16] Payne, W.G., Ko, F., Anspaugh, S., Wheeler, C.K., Wright, T.E. and Robson, M.C. (2006) Down-Regulating Causes of Fibrosis with Tamoxifen: A Possible Cellular/Molecular Approach to Treat Rhinophyma. Annals of Plastic Surgery, 56, 301-305. http://dx.doi.org/10.1097/01.sap.0000199155.73000.2f

[17] Dickens, T.A. and Colletta, A.A. (1993) The Pharmacological Manipulation of Members of the Transforming Growth Factor-Beta Family in the Chemo-Prevention of Breast Cancer. BioEssays, 15, 71-74. http://dx.doi.org/10.1002/bies.950150111

[18] Pollock, M., Constantino, J., Polychronakos, C., et al. (1990) Effect of Tamoxifen on Serum Insulin-Like Growth Factor-1 Levels in Stage 1 Breast Cancer Patients. Journal of the National Cancer Institute, 82, 1693-1697. http://dx.doi.org/10.1093/jnci/82.21.1693

[19] Mancoll, J.S., Zhao, J., McCauley, R.L. and Phillips, L.G. (1996) The Inhibitory Effect of Tamoxifen on Keloid Fibroblasts. Surgical Forum, 47, 718-720.

[20] Chau, D., Mancoll, J.S., Lee, S., et al. (1998) Tamoxifen Downregulates TGF-[Beta] Production in Keloid Fibroblasts. Annals of Plastic Surgery, 40, 490-493. http://dx.doi.org/10.1097/00000637-199805000-00008

[21] Hu, D., Hughes, M.A. and Cherry, G.W. (1998) Topical Tamoxifen: A Potential Therapeutic Regimen in Treating Excessive Dermal Scarring? British Journal of Plastic Surgery, 51, 462-469. http://dx.doi.org/10.1054/bjps.1997.0100

[22] Dvorak, H.F. (1986) Tumors: Wounds That Do Not Heal. The New England Journal of Medicine, 315, 1650-1659. http://dx.doi.org/10.1056/NEJM198612253152606

[23] Bell, E., Ivarsson, B. and Merrill, C. (1979) Production of a Tissue-Like Structure by Contraction of Collagen Lattices by Human Fibroblasts of Different Proliferative Potential in Vitro. Proceedings of the National Academy of Sciences of the United States of America, 76, 1274-1278. http://dx.doi.org/10.1073/pnas.76.3.1274

[24] Ehrlich, H.P., Buttle, D.J. and Bernanke, D.H. (1989) Physiological Variables Affecting Collagen Lattice Contraction by Human Dermal Fibroblasts. Experimental and Molecular Pathology, 50, 220-229. http://dx.doi.org/10.1016/0014-4800(89)90033-6

[25] Germain, L., Jean, A., Auger, F.A. and Garrel, D.R. (1994) Human Wound Healing Fibroblasts Have Greater Contractile Properties than Dermal Fibroblasts. Journal of Surgical Research, 57, 268-273. http://dx.doi.org/10.1006/jsre.1994.1143

[26] Ehrlich, H.P. and Rajaratnam, B.M. (1990) Cell Locomotion Forces versus Cell Contraction Forces for Collagen Lattice Contraction: An in Vitro Model of Wound Contraction. Tissue and Cell, 22, 407-417. http://dx.doi.org/10.1016/0040-8166(90)90070-P

[27] Kuhn, M.A., Moffitt, M.R., Smith, P.D., Lyle, W.G., Ko, F., Meltzer, D.D. and Robson, M.C. (2000) Silicone Sheeting Decreases Fibroblast Activity and Downregulates TGF- $\beta_{2}$ in Hypertrophic Scar Model. International Journal of Surgical Investigation, 2, 467-474.

[28] Simman, R., Alani, H. and Williams, F. (2003) Effect of Mitomycin C on Keloid Fibroblasts: An in Vitro Study. An- 
nals of Plastic Surgery, 50, 71-76. http://dx.doi.org/10.1097/00000637-200301000-00012

[29] Bailey, J.N., Waite, A.E., Clayton, W.J. and Rustin, M.H. (2007) Application of Topical Mitomycin C to the Base of Shave-Removed Keloid Scars to Prevent Their Recurrence. British Journal of Dermatology, 156, 682-686. http://dx.doi.org/10.1111/j.1365-2133.2006.07714.x

[30] Hendricks, T., Martens, M.F., Huyben, C.M. and Wobbes, T. (1993) Inhibition of Basal and TGF Beta-Induced Fibroblast Collagen Synthesis by Antineoplastic Agents. Implications for Wound Healing. British Journal of Cancer, 67, 545-550. http://dx.doi.org/10.1038/bjc.1993.100

[31] Naeini, F.F., Najafian, J. and Ahmadpour, K. (2006) Bleomycin Tattooing as a Promising Therapeutic Modality in Large Keloids and Hypertrophic Scars. Dermatologic Surgery, 32, 1023-1029.

[32] Espana, A., Solano, T. and Quintanilla, E. (2001) Bleomycin in the Treatment of Keloids and Hypertrophic Scars by Multiple Needle Punctures. Dermatologic Surgery, 27, 23-27.

[33] Aggarwal, H., Saxena, A., Lubana, P.S., Mathur, R.K. and Jain, D.K. (2008) Treatment of Keloids and Hypertrophic Scars Using Bleomycin. Journal of Cosmetic Dermatology, 7, 43-49. http://dx.doi.org/10.1111/j.1473-2165.2008.00360.x

[34] Choi, H.S., Savard, C.E., Choi, J.W., Kuver, R. and Lee, S.P. (2007) Paclitaxel Interrupts TGF- $\beta 1$ Signaling between Gallbladder Epithelial Cells and Myofibroblasts. Journal of Surgical Research, 141, 183-191. http://dx.doi.org/10.1016/j.jss.2006.12.558

[35] Liu, X., Zhu, S., Wang, T., et al. (2005) Paclitaxel Modulates TGF $\beta$ Signaling in Scleroderma Skin Grafts in Immunodeficient Mice. PLoS Medicine, 2, e354. http://dx.doi.org/10.1371/journal.pmed.0020354

[36] Jemec, B., Linge, C., Grobbelaar, A.O., Smith, P.J., Sanders, R. and McGrouther, D.A. (2000) The Effect of 5-Fluorouracil on Dupuytren Fibroblast Proliferation and Differentiation. Chirurgie de la Main, 19, 15-22. http://dx.doi.org/10.1016/S1297-3203(00)73455-X

[37] Uppal, R.S., Khan, U., Kakar, S., Talas, G., Chapman, P. and McGrouther, D.A. (2001) The Effect of a Single Dose of 5-Fluorouracil on Keloid Scars: A Clinical Trial of Timed Wound Irrigation after Extralesional Excision. Plastic \& Reconstructive Surgery, 108, 1218-1224. http://dx.doi.org/10.1097/00006534-200110000-00018

[38] Gupta, S. and Karlra, A. (2002) Efficacy and Safety of Intralesional 5-Fluorouracil in the Treatment of Keloids. Dermatology, 204, 130-132. http://dx.doi.org/10.1159/000051830

[39] Bulstrode, N.W., Mudera, V., McGrouther, D.A., Grobbelaar, A.O. and Cambrey, A.D. (2005) 5-Fluorouracil Selectively Inhibits Collagen Synthesis. Plastic \& Reconstructive Surgery, 116, 209-221. http://dx.doi.org/10.1097/01.PRS.0000169701.16509.D6

[40] Occleston, N.L., Daniels, J.T., Tarnuzzer, R.W., et al. (1997) Single Exposures to Antiproliferatives: Long-Term Effects on Ocular Fibroblast Wound Healing Behavior. Investigative Ophthalmology \& Visual Science, 38, 1998-2007.

[41] Ruffy, M.B., Kunnavatana, S.S. and Koch, R.J. (2006) Effects of Tamoxifen on Normal Human Dermal Fibroblasts. Archives of Facial Plastic Surgery, 8, 329-332. http://dx.doi.org/10.1001/archfaci.8.5.329

[42] Mikulec, A.A., Hanasono, M.M., Lum, J., Kadleck, J.M., Kita, M. and Koch, R.J. (2001) Effect of Tamoxifen on Transforming Growth Factor Beta1 Production by Keloid and Fetal Fibroblasts. Archives of Facial Plastic Surgery, 3, 111-114. http://dx.doi.org/10.1001/archfaci.3.2.111

[43] Daniels, J.T., Occleston, N.L., Crowston, J.G. and Khaw, P.T. (1999) Effects of Antimetabolite Induced Cellular Growth Arrest on Fibroblast-Fibroblast Interactions. Experimental Eye Research, 69, 117-127. http://dx.doi.org/10.1006/exer.1999.0684

[44] Seo, S.H. and Sung, H.W. (2012) Treatment of Keloids and Hypertrophic Scars Using Topical and Intralesional Mitomycin C. Journal of the European Academy of Dermatology and Venereology, 26, 634-638. http://dx.doi.org/10.1111/j.1468-3083.2011.04140.x

[45] Jones, C.D., Guiot, L., Samy, M. and Tehrani, H. (2015) The Use of Chemotherapeutics for the Treatment of Keloid Scars. Dermatology Reports, 7, 5880. http://dx.doi.org/10.4081/dr.2015.5880

[46] Manca, G., Pandolfi, P., Gregorelli, C., Cadossi, M. and de Terlizzi, F. (2013) Treatment of Keloids and Hypertrophic Scars with Bleomycin and Electroporation. Plastic and Reconstructive Surgery, 132, 621-630e. http://dx.doi.org/10.1097/PRS.0b013e3182a053c8

[47] Goldan, O., Weissman, O., Regev, E., Haik, J. and Winkler, E. (2008) Treatment of Postdermabrasion Facial Hypertrophic and Keloid Scars with Intralesional 5-Fluorouracil Injections. Aesthetic Plastic Surgery, 32, 389-392. http://dx.doi.org/10.1007/s00266-007-9109-3

[48] Smith, P.D., Mosiello, G., Deluca, L., Ko, F., Maggi, S. and Robson, M.C. (1999) TGF- $\beta_{2}$ Activates Proliferative Scar Fibroblasts. Journal of Surgical Research, 82, 319-323. http://dx.doi.org/10.1006/jsre.1999.5580

[49] Kontochristopoulos, G., Stefanaki, C., Panagiotopoulos, A., Stefanaki, K., Argyrakos, T., Petridis, A. and Katsambas, 
A. (2005) Intralesional 5-Fluorouracil in the Treatment of Keloids: An Open Clinical and Histopathologic Study. Journal of the American Academy of Dermatology, 52, 474-479. http://dx.doi.org/10.1016/j.jaad.2004.09.018

[50] Nanda, S. and Reddy, B.S. (2004) Intralesional 5-Fluorouracil as a Treatment Modality of Keloids. Dermatologic Surgery, 30, 54-57. 